\title{
Sexism and League of Legends: NFR aesthetic analyses
}

\author{
Luiz Paulo Carvalho ${ }^{1}$, Claudia Cappelli ${ }^{12}$ \\ 1 PPGI- Programa de Pós-Graduação em Informática \\ 2 DIA - Departamento de Informática Aplicada \\ Universidade Federal do Estado do Rio de Janeiro (UNIRIO) \\ Av. Pasteur, 296 - Urca - Cep 22290-240 - Rio de Janeiro - RJ - Brasil \\ \{luiz.paulo.silva, claudia.cappelli\}@uniriotec.br
}

\begin{abstract}
Sexism is a prejudiced behavior or discrimination based on sex or gender existent in digital games environment and systems requirements can consciously, or not, enable it. In a previous work, the world's most played and disseminated online digital game presented a great number of sexism cases reported via a questionnaire referring to sexism in digital games, being highlighted as most negatively cited by respondents. This work aims to provide an understanding about this fact by analyzing the technical aspect of the nonfunctional aesthetic system requirements of the game to validate the presence of sexism. Concluding, sexism is present in the analyzed aspect of the game.
\end{abstract}

\section{Introduction}

Digital games are traditionally a male-dominant space, "an activity created by men and for men". Women are generally poorly represented in video games content, promoting the normalization and encouragement of sexual harassment [Fox and Tang, 2013], contributes to consolidate these ideas in the social development of children and teenagers, influencing their attitudes and behaviors towards women [Dill et al., 2008].

Gamers interact with digital games through functions (walk; jump; shoot; sit etc.). In addition to functions games have quality properties, or non-functional requirements (NFR) such as security, accuracy, usability, intuitiveness, aesthetics etc. [Wieringa, 2014]. Some need assurance by functional requirements (FR), such security is assured by password validators; while others do not, like colors.

Female players may develop dissatisfaction with themselves when not meeting those expectations imposed by the female character's appearance [Near, 2013] and question their abilities compared to a man [Morawitz, 2007], causing them to self-select out of such environment [Fox and Tang, 2013], which maintains the predominance of men and reinforces that games, and game development, is a male activity.

Sustainability can be defined as "development that meets the needs of the present without compromising the ability of future generations to meet their own needs", not only encompassing natural resources but also social development [WCED, 1992]. Pursuing social justice is one way to ensure the well-being and quality of life of present and future generations [UNESCO, 2002].

In Carvalho et al., (2017), a questionnaire was conducted with Information Systems (IS) students of a Brazilian public university to find out if sexism was present 
in the respondent's lives regarding digital games scenario. League of Legends (LoL) had relevant negative repercussion by the interviewees, with more reports of sexism cases. The goal is to analyze the game in attempt to validate the answers, since it has a great number of players and great influence, impacting social sustainability world-wide.

This work does not deepen the questionnaire or its results, this step has already been done in Carvalho et al. (2017). As results: (i) Sexism, positive or negative, affects mainly women; (ii) All questionnaire respondents believe that there is sexism in digital games; (iii) Most respondents, both men and women, believe that education is a way to solve the sexism behavior, in line with UNESCO (2002); (iv) LoL is the most quoted game associated to sexism in digital games. A word cloud was generated using the questionnaire responses and LoL (League, Legends, LoL) appeared in a relevant number, as illustrated by Figure 1.

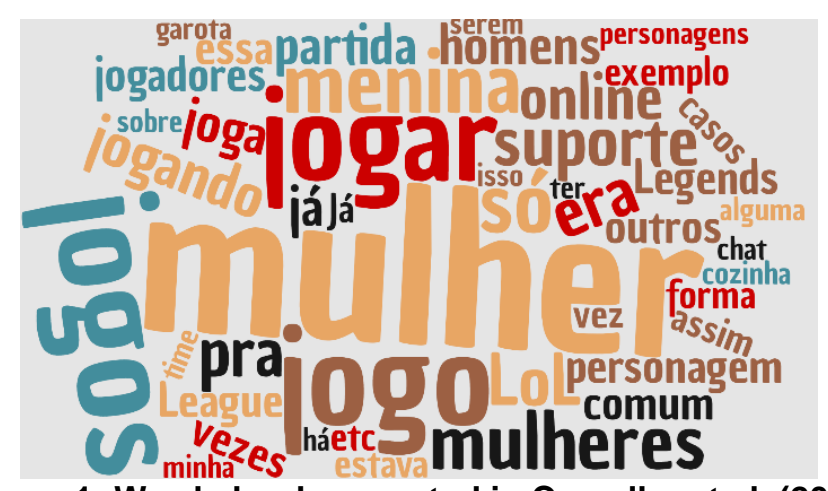

Figure 1: Word cloud presented in Carvalho et al. (2017)

This work analyzes the aspects of system non-functional requirements (NFR) qualities of the game, reportedly related to sexism in previous literature, aiming to answer the following question "Does League of Legends digital game enable sexist in its non-functional requirements?"

This paper is organized as follows, Section 2 presents relevant concepts; Section 3 presents the analyses; Section 4, the respective results; Section 5 concludes this work.

\section{Theoretical foundation}

\subsection{Sexism in functional or non-functional requirements}

Plenty of work deals with the association between sexism and digital games [Ivory, 2006; Morawitz, 2007; Downs and Smith, 2010; Martins et al., 2011; Downs and Smith, 2010; Stermer and Burkley, 2015; Bègue et al., 2017]. Carvalho et al. (2017), work preceding this, presented the relation between ethics in the development of systems and sexism; it is responsibility of the creators (not just developers) of the game to preserve, directly or indirectly, social sustainability.

Requirements are treatment of goals [Wieringa, 2014]. Functional requirements (FR) are the direct use of services provided by the game [Chung et al., 2000]. If the game foster stereotypes of social roles based on sex in its functions, sexism is implemented. For example, if the game does not allow the user to dress his male character with high heels or skirts, sexism occurs. Non-functional requirements (NFR) are implemented from quality properties; subjective, depending on who interacts with it; determine how the systems will operate; and are harder to deal with [Chung et 
al., 2000]. NFR impact directly or indirectly; consciously or unconsciously in the respective system. Sexism, as a negative aspect and in most cases, result from indirect unconscious properties. Some system aspects enable sexism without that goal (indirectly) and without the clarity of the behavior in question (unconsciously).

One social responsibility that impacts organizations is legal [Carroll, 2001]. In specific contexts sexism is mandatory by law. A LoL championship in Iran excluded almost all the female champions from the game to comply with Iranian laws ${ }^{1}$.

The current scenario of digital games reflects a social dispute for respect, inclusion and representativeness. Women are presented, constantly questioned about their right to occupy this space. Sexism is one of the discriminations suffered by female players in this environment, by the lack of representativeness, contributing negatively to the maintenance of women in the digital games scenario [Fox and Tang, 2013].

In Social cognitive theory [Bandura, 2001] people are exposed to models that teach them about the symbolic representation of the world, making them believe that those signs, or their absence, is the pattern to be followed. Cultivation theory [Gerbner et al., 2002] proposes that the understanding and perception of social reality is influenced by the repeated exposure of media, so the socially accepted is what the media constructs expose repeatedly to us, or, on the other hand, do not expose.

Most women are not necessarily thin, physically weak, dress partially naked, wear high heels (especially in adventures and fighting context), have big breasts or buttocks, strong legs etc. When systems represent a female character with all of these characteristics they representatively exclude the greater share of women, discarding them from reality. For example, "I am not thin, I will never be included" or "Look how the boobs of all these characters are big, are mine a problem?".

\subsection{League of Legends}

A rich Wiki ${ }^{2}$ was used as game data source, synthesizing the game information, also a source widely disseminated by the community. The game version analyzed was 8.13, released on 06/27/2018, on BR server (Brazilian server).

LoL is a digital game, included in the Mobile Online Battle Arena (MOBA) genre, also being an electronic sport (eSports). It can be played in single or multiplayer mode online. There are ten server environments around the world; Brazil has its own server, BR. The game is maintained by Riot Games and was released in October $2009^{2}$.

LoL is the game with the largest number of online players in the world, in 2016 Riot Games announced that the game had a hundred million active monthly players ${ }^{3}$. According to SuperData, a company that provides market intelligence, LoL generates US $\$ 150$ million per month and US\$1.8 billion annually ${ }^{4}$.

There are one hundred forty-one playable characters in the game. Every character has at least one skin besides the classic one. Skins are cosmetic modifications that players can enable to one or more base characters, some characters have more than

\footnotetext{
${ }^{1} \mathrm{https}: / /$ kotaku.com/female-league-of-legends-characters-banned-at-an-irania-1213754332

$2 \mathrm{http} / / /$ leagueoflegends.wikia.com/

${ }^{3} \mathrm{https}: / /$ goo.gl/7Ttxet

${ }^{4}$ https://goo.gl/sb3uob
} 
ten skins while others just one. Besides the characters, called "champions", there are dozens of items and some rotative game modes and scenarios.

\section{Case Study: League of Legends non-functional requirement analysis}

NFR is related to quality, not function [Wieringa, 2014]. Character abilities are individual to each and gender independent. There is no functionally better female than male, or vice versa. Gender choice when developing characters is objectively Riot responsibility (FR), visual aesthetic design of characters too (NFR).

Sommerville (2007) lists several categories and subcategories for non-functional requirements such as efficiency, usability, delivery, standards, legal, geographical, etc. In this work just one is analyzed and quantitatively evaluated, visual aesthetic design.

There are forty-nine female characters and ninety-one male characters. Only one agender, Bard. Kindred was developed as two characters, but effectively only the lamb (female) is playable, not the wolf (male). Figure 2 shows Bard and Kindred.

A Google research with time criteria and STRING "sexism league legends" has shown that since July 2011 (first found) is produced material related to sexism in visual design of LoL humanized female characters ${ }^{5}$. In this matter, a comment related to "boobs" in the Riot Games official forum said: "We need moar boobs" ${ }^{5}$. LoL does not propose, directly or consciously, to deliver any value related to sensuality or eroticism.

Answers from the previous work, Carvalho et al. (2017), were related to sexism NFR, mainly aesthetic. Few excerpts: "[...] clothes that female characters usually use ("revealing") and body shape (Playboy magazine standard) [...]"; "[...] ridiculous characterization of the female characters, who are practically naked or if not always hypersexualized, with big breasts and ass, skinny (playboy bunny style) and even wearing high heels (which would be totally incompatible with fight / adventure) [...]"; and "[...] female characters have real armor, not an iron bikini $[\ldots]$ ".

To confirm if sexism aesthetics NFR characteristics was present in the game, all the visual representations of characters was evaluated and associated with sexist characteristics presented in literature and questionnaire answers [Carvalho et al., 2017]. Both genders are analyzed, a sexism characteristic is dichotomy between stereotypes.

Theories in Section 2.1 was used to determine elements to be analyzed. Excluded: undead; children; characters with human aspects, but very animalistic (e.g., Kindred ${ }^{2}$ ); and distant of human physiology (e.g., yordles ${ }^{2}$ ). Included: Animalistic, with just one or two body parts different from human shape, close to human physiology. According to these two theories players would not create a physical representative relationship with these characters. Criteria: (1) cleavage and legs, (2) bare-arms, (3) bare-belly, (4) vestment [Downs and Smith, 2010]; (5) body shape, (6) explicit strength [Martins et al., 2011]; (7) "boobplate", (8) shoes, (9) armor (10) coherence with character proposal [Carvalho et al., 2017].

Only the classic graphical representations of the characters were analyzed and evaluated, because they are available to players as soon as they access the game for the

\footnotetext{
${ }^{5}$ https://goo.gl/vdyGCs
} 
first time. Skins, cosmetic NFR modifications, need to be bought or acquired after stipulated goals are achieved playing the game.

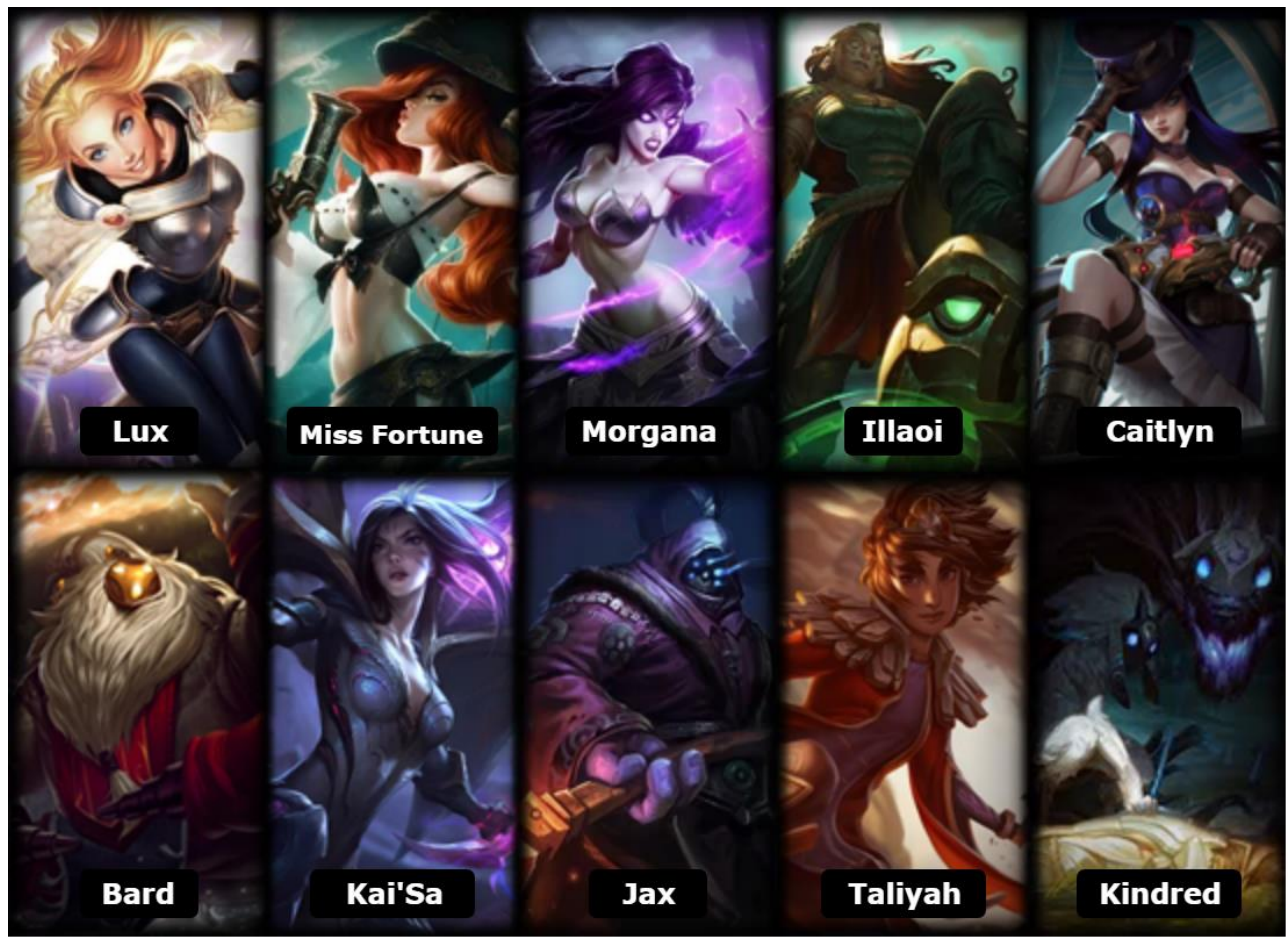

Figure 2: League of Legends playable characters examples ${ }^{26}$

After exclusion by the criteria presented above and separation of the classic skins, the research was conducted using eighty-eight elements. Of them, forty females and forty-eight males and some of them are illustrated in Figure 2.

\section{Results}

Table 1 presents the result of the specific criteria analyzed. Totaling, they are $45.5 \%$ female and $54.5 \%$ male. Figure 2 shows some of the characters mentioned in the results.

Cleavage and legs (1), 266\% more males do not expose their body. The function of the cleavage is to enhance and value the breast. Only females explicitly expose their legs to strengthen their silhouette and gender. The males expose their pectoral to expose virility, no thin character exposes it. Arms (2), no relevant discrepancy. Males explicitly expose their arms to expose muscles. Belly (3), no relevant discrepancy. Vestment (4), no discrepancy. Again, the half-naked males reinforce their muscles and virility. Zyra is the only naked character in the game, what looks like her outfit is actually her skin. Even then she wears high heels.

\footnotetext{
${ }^{6}$ All image rights reserved to Riot Games, Inc. League of Legends $@$ Riot Games, Inc. Riot Games allow the use of its intellectual property in compliance with terms in https://www.riotgames.com/en/legal.
} 


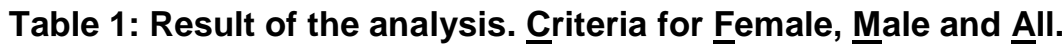

\begin{tabular}{|c|c|c|c|c|c|c|c|c|c|c|c|c|c|c|c|}
\hline C & Category & $\mathbf{F}$ & $\%$ & M & $\%$ & $\mathbf{A}$ & $\%$ & C & Category & $\mathbf{F}$ & $\%$ & $\mathbf{M}$ & $\%$ & A & $\%$ \\
\hline \multirow{4}{*}{1} & No & 12 & 30,0 & 32 & 66,7 & 44 & 50,0 & 5 & Thin & 39 & 97,5 & 43 & 78,8 & 82 & 93,2 \\
\hline & Cleavage (C.) & 15 & 37,5 & 1 & 2,1 & 16 & 18,2 & \multirow{3}{*}{6} & Beefy & 2 & 5,0 & 43 & 89,6 & 45 & 51,1 \\
\hline & C. and legs & 13 & 32,5 & 0 & 0,0 & 13 & 14,8 & & Weakly & 38 & 95,0 & 2 & 4,2 & 40 & 45,5 \\
\hline & Bare pectoral & 0 & 0,0 & 15 & 31,3 & 15 & 17,0 & & Unknown & 0 & 0,0 & 3 & 6,3 & 3 & 3,4 \\
\hline \multirow{3}{*}{2} & Yes & 10 & 25,0 & 16 & 33,3 & 26 & 29,5 & \multirow{3}{*}{7} & Yes & 9 & 22,5 & 0 & 0,0 & 9 & 10,2 \\
\hline & Partial & 15 & 37,5 & 11 & 22,9 & 26 & 29,5 & & Yes (armor) & 9 & 90,0 & 0 & 0,0 & 9 & 10,2 \\
\hline & No & 15 & 37,5 & 21 & 43,8 & 36 & 40,9 & & No & 31 & 77,5 & 48 & 100,0 & 79 & 89,8 \\
\hline \multirow{3}{*}{3} & Yes & 11 & 27,5 & 11 & 22,9 & 22 & 25,0 & \multirow{3}{*}{8} & Yes & 10 & 25,0 & 0 & 0,0 & 10 & 11,4 \\
\hline & Partial & 4 & 10,0 & 6 & 12,5 & 10 & 11,4 & & No & 25 & 62,5 & 48 & 100,0 & 73 & 83,0 \\
\hline & No & 25 & 62,5 & 31 & 64,6 & 56 & 63,6 & & No legs & 5 & 12,5 & 0 & 0,0 & 5 & 5,7 \\
\hline \multirow{4}{*}{4} & Total & 1 & 2,5 & 3 & 6,3 & 4 & 4,5 & & Heavy & 2 & 5,0 & 7 & 14,6 & 9 & 10,2 \\
\hline & Complete & 28 & 70,0 & 30 & 62,5 & 58 & 65,9 & & Light & 8 & 20,0 & 12 & 25,0 & 20 & 22,7 \\
\hline & Half-naked & 10 & 25,0 & 15 & 31,3 & 25 & 28,4 & & No & 30 & 75,0 & 28 & 58,3 & 58 & 65,9 \\
\hline & Naked & 1 & 2,5 & 0 & 0,0 & 1 & 1,1 & & Unknown & 0 & 0,0 & 1 & 2,1 & 1 & 1,1 \\
\hline
\end{tabular}

Body shape (5), only Illaoi is a non-thin female character. The thinness is not exclusively related to female character, the majority of male are also thin (78.8\%). Explicit strength (6), discrepancy is clear. Characters who expose absolutely nothing of the body are males (Jax, Nautillus and Zed), and they are clearly defined as males, despite the cover of the clothes. $4 \%$ of males are explicitly thin and $90 \%$ are muscular, regardless of the character's proposal, such as mages. Illaoi and Sejuani are the only muscular females.

"Boobplate" (7), 90\% of females wearing armor show the same with "breast compartment". No male wears armor that explicitly salient his chest. Shoes (8), only female wear heels (high or not) and 25\% wear high heels, regardless of character's proposal. Armor (9), discrepancy is clear. Heavy covers all body, light just some parts. Not only in number, design and appearance of armors are explicitly different.

Coherence with the character proposal (10) is a subjective and creative aspect. A character designer from Riot Games has officially stated: "The key is the character's looks HAVE TO MAKE SENSE. [...] The way Caitlyn looks makes no sense for a prim and proper sheriff" 7 . Caitlyn is identified as "The Sheriff of Piltover" 2. Therefore, there is no guarantee of epistemological quality in the characters design.

\section{Conclusion and discussion}

This work contributes with a structured systematic quantitative review of the aesthetic NFR elements of humanized characters from the world-wide game League of Legends. Referring to aesthetic NFR, LoL enables sexism. Criteria 2, 3, 4 do not reinforce sexist behavior; criteria 1 and 5 to 10 reinforce. Corroborating criticisms that graphic artists make about the game, such as the League of Sexism ${ }^{8}$ page. On criterion 6, feminine figures are constructed with anatomical drawing to emphasize physical attributes considered feminine, like breasts; buttocks; thinness etc. even if they are non-real ${ }^{9}$.

\footnotetext{
${ }^{7}$ https://nexus.leagueoflegends.com/en-us/2017/04/ask-riot-the-sexy-factor/

${ }^{8} \mathrm{http}: / /$ leagueofsexism.tumblr.com/

${ }^{9} \mathrm{https}$ //evilgeeks.com/2015/06/20/female-objectification-in-games-league-of-sexism-a-rant/
} 
In Figure 2, Lux and Miss Fortune anatomy are realistically impossible. Building female characters with emphasis on sexual appeal rather than focusing on their context, history, or role is frequent in digital games [Ivory, 2006]. Difference between female and male thinness is inconsistent with the game's combat theme. Females classified as "Fighter" are physically frail and run in high heels; males are muscular, even in traditionally weak classes, such as "Mages".

In April 2018, for the first time a Lead Producer of Champions (REAV3) spoke about sexism and hyper sexualization, stated officially about Kai'Sa (shown in Figure 2) exaggerated cleavage: "We'll be the first to admit that Kai'Sa's neckline didn't land well with players and that it made her look unnecessarily sexualized, even if it that wasn't the intention. [...] Even though we had good intentions, we could've done better, and in the future, we will be even more conscious of these decisions" ${ }^{10}$.

Riot Games decreased the sexist behavior in its three latest female characters. Illaoi, Taliyah and the Irelia update ${ }^{2}$ extended the diversity of female aesthetics in the game. Illaoi is the only bulky and muscular female. Taliyah has no graphic appeal to her breasts or buttocks; her face is not standardized ${ }^{8}$ and shows no sensual element, not needing any sexist element to be explicitly female.

Success of Gamification in Information Systems is growing [Schawa, 2016]. To ensure social sustainability developers should avoid sexism in system requirements, among them, aesthetic. Looking for references to visual design of characters they can find, or already know, LoL, the most played digital game. This work quantitatively exposes some sexist elements in LoL. One of the current IS Great Challenges in Brazil (GranDSI-BR) is the conscious and ethic systemic view of systems impact on the organization of life and society [Boscarioli et al., 2017].

As limitations, not all skins (only classic ones) and not all sexism aspects were analyzed; and only aesthetic NFR. Certain points are subjective and context-dependent, for example, concept of sexualization in Brazil is different from Iran or Japan.

As future work, analysis on sexism and LoL: FR details; social aspects (external and internal community); organizational aspects (how Riot Games deals with sexism as a company); more sexism aspects; and more skins. Table 1 analyzed data can be openly found, manipulated and analyzed online ${ }^{11}$.

\section{References}

All online references used in this work was available in 08/08/2018.

Bandura, A., "Social cognitive theory of mass communication" (2001) Media Psychol. 2: 265-299.

Boscarioli, C., Araujo, R., Maciel, R., "GranDSI-BR - Grand Research Challenges in Information Systems in Brazil 2016-2026" (2017). Special Committee on Information Systems (CE-SI), Brazilian Computer Society (SBC).

Bègue, L., Sarda, E., Gentile, D. A., Bry, C., Roché, S., "Video Games Exposure and Sexism in a Representative Sample of Adolescents" (2017) Front. Psychol. 31: 466.

\footnotetext{
${ }^{10} \mathrm{https}: / /$ nexus.leagueoflegends.com/en-us/2018/04/ask-riot-kaisas-v-neck-suit/

${ }^{11} \mathrm{https} / / /$ goo.gl/GwKn53
} 
Carroll, A., "The Pyramid of Corporate Social Responsibility: Toward the Moral Management of Organizational Stakeholders" (1991). Business Hor. 34 (4): 39-48.

Carvalho, L. P., Cappelli, C., Santoro, F., "Sexism and Digital Games, a Brazilian University Exploratory Research.” (2017) Joinville, IHC CAPA.

Chung, L., Nixon, B., Yu, E., Mylopoulos, J., "Non-functional Requirements in Software Engineering" (2000). Netherlands, Kluwer Academic Publishers.

Dill, K., Brown, B., Collins, M., "Effects of exposure to sex-stereotyped video game characters on tolerance of sexual harassment." (2008) Journal of Experimental Social Psychology 44 (5): 1402-1408.

Downs, E., Smith, S., "Keeping abreast of hypersexuality: a video game character content analysis" (2010) Sex Roles 62: 721-733. doi: 10.1007/s11199-009-9637-1.

Fox, J., Tang, W. T., "Sexism in online video games: The role of conformity to masculine norms and social dominance orientation." (2014) Computers in Human Behavior 33: 314-320.

Gerbner, G., Gross, L., Morgan, M., Signorelli, N., Shanahan, J. "Growing up with television: Cultivation processes" (2002) Media effects: Advances in theory and research 2: 43-67.

Ivory, J. D., 'Still a man's game: gender representation in online reviews of video games" (2006) Mass Comm. Soc. 9: 103-114.

Martins, N., Williams, D., Ratan, R., Harrison, K., “A content analysis of female body imagery in video games" (2011) Sex Roles 61: 824-836.

Morawitz, E., "Effects of the sexualization of female characters in video games on gender stereotyping, body esteem, self-objectification, self-esteem, and self-efficacy." (2007) Arizona, The University of Arizona.

Near, E., "Selling gender: Associations of box art representation of female characters with sales for teen-and mature-rated video games." (2013) Sex roles 68 (3): 252-269.

Sommerville, I., "Software Engineering" (2015) Ed. 10. Pearson.

Stermer, P., Burkley, M., "Sex-box: exposure to sexist video games predicts benevolent sexism" (2015). Psychol. Pop. Media Cult. 4: 47-55.

Swacha, J., "Gamification in Enterprise Information Systems: What, Why and How" (2016). ACSIS, Vol. 8: 1229-1233.

UNESCO. "Education for Sustainability. From Rio to Johannesburg: Lessons learnt from a decade of commitment" (2002). Available in: https://goo.gl/M3kt2T

Wieringa, R., "Design Science Methodology for Information Systems and Software Engineering" (2014). Berlin, Springer-Verlag.

World Commission on Environment and Development, "Report of the World Commission on Environment and Development: Our Common Future" (1992). Available in: https://goo.gl/vKf5qV 